\title{
Empyème pulmonaire chez un patient schizophrène ayant une mauvaise dentition
}

\author{
Hongru Ren MD, Andrew Walkty MD, Biniam Kidane MD MSc
}

- Citation : CMAJ 2021 November 8;193:E1695. doi : 10.1503/cmaj.202556-f

Voir la version anglaise de l'article ici : www.cmaj.ca/lookup/doi/10.1503/cmaj.202556
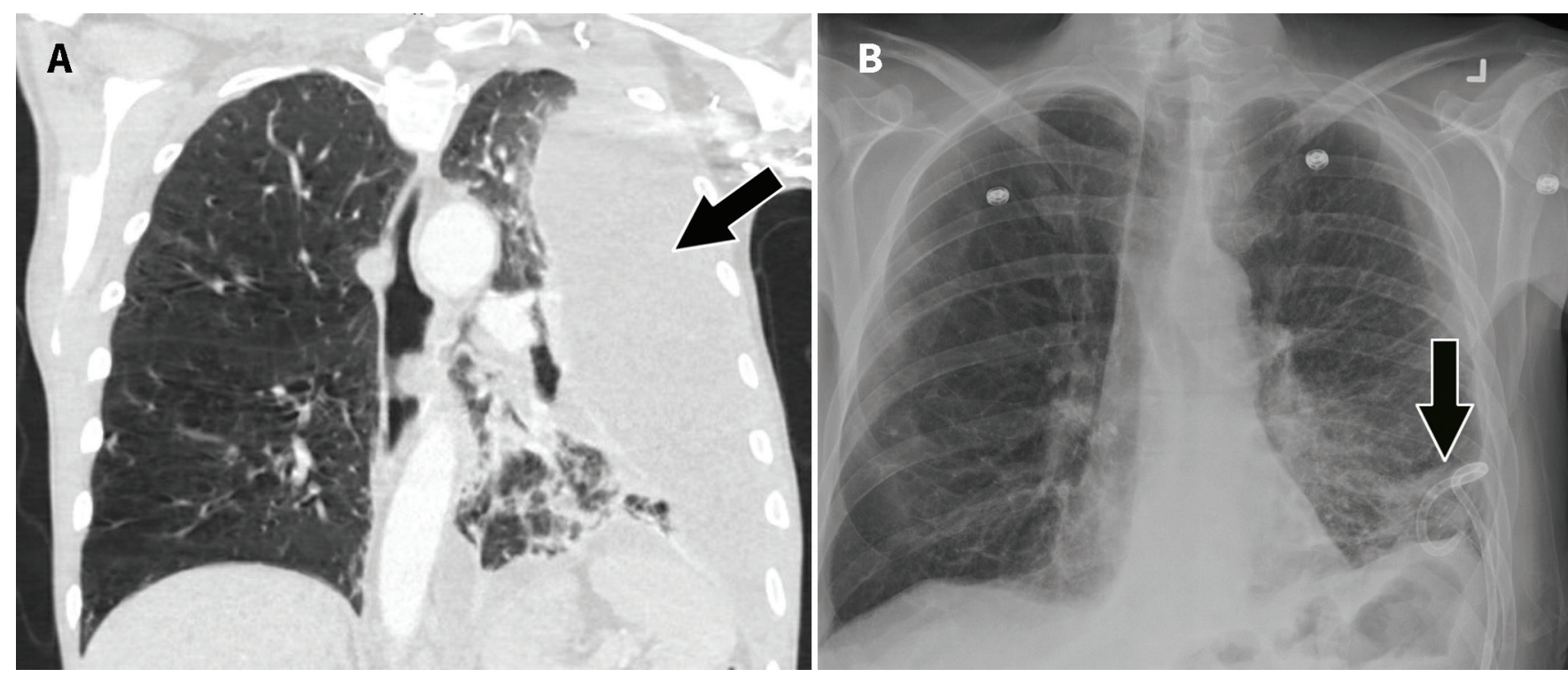

Figure $1:$ A) Tomodensitométrie avec agent de contraste montrant un empyème loculé du côté gauche (flèche) en coupe coronale. B) Radiographie pulmonaire postérieure/antérieure montrant la résolution complète de l'empyème après l'insertion d'un cathéter à queue de cochon (flèche) et l'administration de 3 doses d'activateur tissulaire du plasminogène intrapleural et de dornase alfa, avec poursuite de l'antibiothérapie intraveineuse.

$\mathbf{U}$ n homme de 55 ans ayant des antécédents médicaux de schizophrénie et de troubles convulsivants a consulté à notre hôpital pour un problème subaigu de toux, perte de poids et confusion. Nous l'avons examiné. Il nous a semblé cachectique, il était désorienté et afébrile, mais hémodynamiquement stable. Nous avons noté chez lui plusieurs caries dentaires. La radiographie pulmonaire et la tomodensitométrie ont montré une consolidation au lobe inférieur gauche avec épanchement pleural loculé (figure 1A). L'analyse du liquide pleural a confirmé le diagnostic d'empyème (turbidité, protéines $42 \mathrm{~g} / \mathrm{L}$, ratio des protéines du liquide pleural : protéines sériques 0,8 , lactate déshydrogénase $958 \mathrm{U} / \mathrm{L}$, ratio des protéines du liquide pleural : lactate déshydrogénase sérique 5,5).

La coloration de Gram d'un spécimen de liquide pleural obtenue après 3 jours d'antibiothérapie a révélé la présence de bacilles à Gram négatif. La culture aérobie du liquide pleural n'a révélé la croissance d'aucun agent pathogène; par contre, on a isolé Slackia exigua (bacille à Gram positif) à la culture anaérobie (annexe 1, accessible en anglais au www.cmaj.ca/lookup/ doi/10.1503/cmaj.202556/tab-related-content). Compte tenu des résultats différents entre la coloration de Gram et la culture du liquide pleural, nous avons diagnostiqué une infection polymicrobienne probablement consécutive à l'aspiration.

Nous avons administré un traitement fibrinolytique intrapleural par cathéter à queue de cochon pour faciliter le drainage de l'empyème (figure 1B). Le patient a mené à terme un cycle de 3 semaines d'amoxicilline-clavulanate par voie orale, il s'est rétabli, puis a été soumis à un traitement dentaire définitif après une mobilisation concertée de notre équipe pendant plusieurs mois en collaboration avec sa travailleuse sociale, son foyer d'hébergement, le curateur public, le mandataire et le service des soins dentaires ambulatoires.

L'empyème peut survenir après une aspiration. Chez notre patient, les facteurs de risque à l'égard de l'aspiration et de 
l'empyème qui en résulte incluaient mauvaise dentition, utilisation d'antipsychotiques et antécédents de convulsion. S. exigua est un bacille anaérobie ne formant pas de spores qui fait partie du microbiote buccal humain ${ }^{1}$. On l'associe le plus souvent à des infections dentaires, mais il a déjà été isolé dans des cultures provenant de patients porteurs d'infections extrabuccales, telles qu'empyème et abcès pulmonaire, méningite, thrombose du sinus caverneux et abcès intra-abdominal ${ }^{2}$. Les infections à S. exigua ont tendance à être polymicrobiennes; et c'est ce que nous avons présumé chez notre patient. La maladie mentale et la situation socio-économique de ce patient ont été des obstacles à l'obtention d'un traitement dentaire préventif. Ce cas rappelle le rôle important de la mauvaise santé dentaire et de l'aspiration dans la pathogenèse des infections pulmonaires.

\section{Références}

1. Wade WG, Downes J, Dymock D, et al. The family Coriobacteriaceae: reclassification of Eubacterium exiguum (Poco et al. 1996) and Peptostreptococcus heliotrinreducens (Lanigan 1976) as Slackia exigua gen. nov., comb. nov. and Slackia heliotrinireducens gen. nov., comb. nov., and Eubacterium lentum (Prevot 1938) as Eggerthella lenta gen. nov., comb. nov. Int J Syst Bacteriol 1999;49:595-600.

2. Kawasuji H, Kaya H, Kawamura T, et al. Bacteremia caused by Slackia exigua: a report of two cases and literature review. J Infect Chemother 2020;26:119-23.

Intérêts concurrents : Aucun intérêt concurrent n'a été déclaré.

Cet article a été révisé par des pairs.

Les auteurs ont obtenu le consentement du patient.

Affiliations : Départements de médecine interne (Ren) et de microbiologie médicale et infectiologie (Walkty), Faculté de médecine Max Rady, Université du Manitoba; Soins communs Manitoba (Walkty); Section de chirurgie thoracique (Kidane), Département de chirurgie, Faculté des sciences de la santé Rady et Département de santé communautaire (Kidane), Université du Manitoba; Institut manitobain de recherche sur le cancer (Kidane), Winnipeg, Man.

Propriété intellectuelle du contenu : Il s'agit d'un article en libre accès distribué conformément aux modalités de la licence Creative Commons Attribution (CC BY-NC-ND 4.0), qui permet l'utilisation, la diffusion et la reproduction de tout médium à la condition que la publication originale soit adéquatement citée, que l'utilisation se fasse à des fins non commerciales (c.-à-d., recherche ou éducation) et qu'aucune modification ni adaptation n'y soit apportée. Voir : https://creativecommons.org/licenses/by-nc-nd/4.0/deed.fr.

Correspondance : Biniam Kidane, bkidane@hsc.mb.ca

Les images cliniques sont choisies pour leur caractère particulièrement intéressant, classique ou impressionnant. Toute soumission d'image de haute résolution claire et bien identifiée doit être accompagnée d'une légende aux fins de publication. On demande aussi une brève explication (300 mots maximum) de la portée éducative des images, et des références minimales. Le consentement écrit du patient au regard de la publication doit être obtenu avant la soumission. 